\title{
New trends in materials science
}

in the field of materials science where both physics and chemistry are concerned, the leading evolutions in the most recent years are the result of a better awareness by fundamentalists of applicative needs. Many new insights have been given recently by materials scientists to magnetism, non-linear optics or chemical reactivity, among others. Moreover, one may consider that the need for extensive miniaturization, which is of major importance in microelectronics industry and for the progress in information and communication technologies, has been decisive to promote research in the field of nanostructures and low-dimensionality materials.

The various contributions to this issue of Analusis EurJAC illustrate this way of thinking and show how a multidisciplinary approach, coupling chemists and physicists, associated with instrumental developments well suited to the elaboration and characterization of new materials have been determining factors. They allowed one to master the growth of micro- and nano-objects and to obtain model materials, easier to analyze than raw industrial samples but realistic enough for well predicting the properties needed for industrial development.

As far as characterization and properties of nanostructures and low-dimensionality materials are concerned, the main developments in the near future lie in new instrumental possibilities: spatial (and more specifically lateral size), energy and time resolution are nowadays reduced drastically thanks

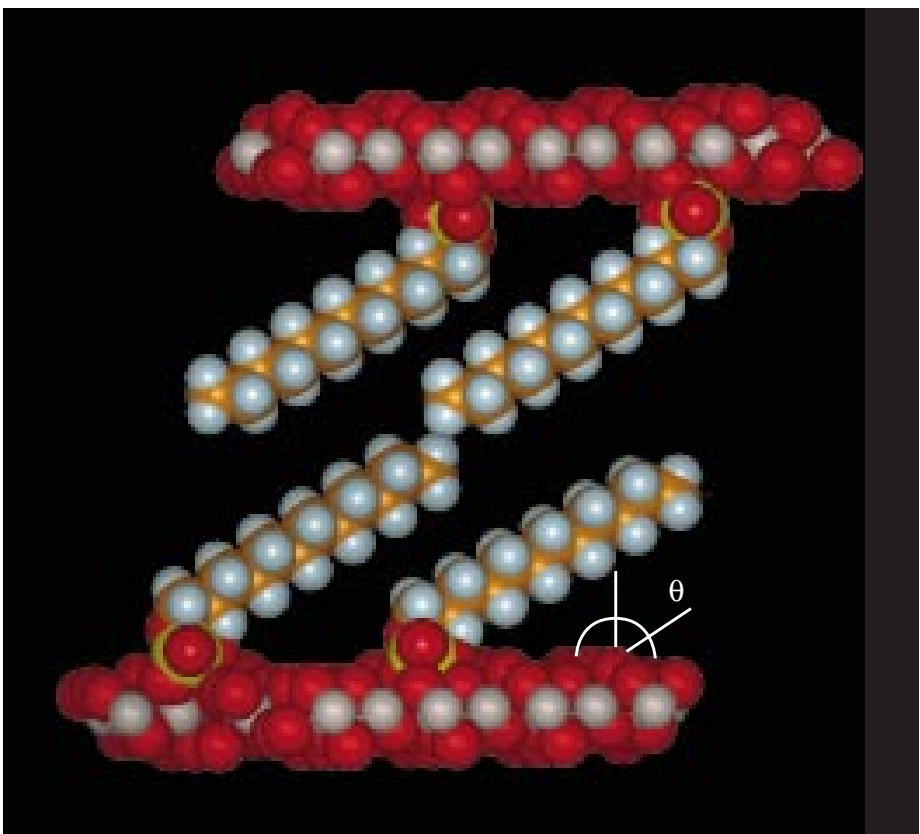

to new experimental techniques or to more classical analytical methods, which are regularly revisited and improved.

The examples given in this issue illustrate the main experimental and theoretical procedures used in several domains of materials science and the contribution of various techniques. The first one deals with "Molecular Optoelectronics". The synthesis and characterization of organic molecules which can have photovoltaic, photorefractive or electroluminescent properties are described. The second one is related to hybrid organic-inorganic layered magnets and shows how chemical engineering can be used to change the magnetic properties of these systems and thus to understand the various models of interaction. The third one is about aggregates in silica based matrices. It shows how metallic or organic nanostructures can be embedded in an organic matrix to control their size and protect them against degradation, in order to improve their optical and magnetic properties. the fourth one describes the growth of self-organized cobalt clusters. Scanning tunnelling microscopy is used at variable temperature to control the morphology and the distribution size of cobalt nanostructures, inducing special magnetic properties. Finally, the fifth one deals with the growth of magnetic ultrathin films and multilayers. It is focused on the several ways of characterizing the crystal structure (long-range or local) in the vicinity of surfaces and interfaces and to correlate it with the macroscopic properties.

Beyond these contributions, it is probably worth emphasizing on some recent results which illustrate these ultimate possibilities in resolution. The first one concerns the atomic resolution obtained by high resolution transmission electron microscopy (HRTEM). Figure 1 shows a high resolution TEM image of nanometric platinum particles obtained from a platinum-rhodium alloy, the size of which is less than $7 \mathrm{~nm}$ in diameter. They are dispersed on high specific area silicon carbide $(\mathrm{SiC})$ and are covered by cerine $(\mathrm{CeO})_{2}[1]$. This structural and morphological characterization on an atomic scale allowed improving the catalytic properties of such materials, through the analysis of the spatial relationships between $\mathrm{Pt}-\mathrm{Rh}$ and $\mathrm{CeO}_{2}$.

Significant progress in the study of spatial variations in the composition and electronic structure of surfaces is now expected thanks to the new techniques of spectromicroscopy which are developed on the third generation synchotron radiation facilities. Synchrotron-radiation scanning photoelectron microscopy combines chemical mapping from selected spots at the surface of materials with photoelectron spectroscopy. In this way the spatial variations in composition and electronic structure at the Ni/Si interfaces have been studied [2]. The combination of a submicron lateral resolution $(\leq 0.15 \mu \mathrm{m})$ with a high resolution in energy $(0.34 \mathrm{eV})$ allowed the characterization of

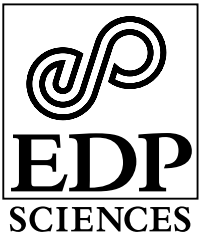

\author{
Coordinated by: Bernard Carrière \\ Institut de Physique et Chimie des Matériaux de Strasbourg \\ UMR 7504, CNRS-Université Louis Pasteur http://www.edpsciences.org 
coexisting silicide phases on the surface. In the same point of wiew, core-level photoelectron spectroscopy (XPS-ESCA) remains a powerful analytical tool despite the difficulties in interpreting core-level shifts and lineshapes with high energy resolution [3].

As far as applications with magnetic devices are concerned, one of the main goals, beyond accurate structural and morphological characterizations of the nanostructures, is to study in real time their magnetic behaviour. Understanding the dynamics of relaxation when probing the magnetization state by laser pulses has lead to a new field called "femtomagnetism". Here, the ultimate time resolution allowed by the new femtosecond laser sources opens potential applications in the field of magneto-optical devices for information recording [4].

Finally, one should emphasize the decisive impact of the cooperation between theory and experiment, either to predict new optical or magnetic properties, related to the reduction in dimensionality, or to perform the modelization of the results, for example for the morphological and structural characterization of the materials, the determination of their electronic structure or the time-resolved study of the time-dependent properties.

\section{References}

1. Benaissa, M.; Pham-Huu, C.; Werckmann, J.; Ledoux, M. J. Catalysis Today 1995, 23, 283.

2. Gregoratti, L.; Günther, S.; Kovac, J.; Marsi, M.; Phanuef, M. J.; Kiskinova, M. Phys. Rev. B 1999, 59, 2018; Gregoratti, L.; Günther, S.,; Kovac, J.; Casalis, L.; Marsi, M.; Kiskinova, M. Phys. Rev. B 1998, 57, R6799.

3. Grupp, C.; Taleb-Ibrahimi, A. J. Electron Spectrosc. Relat. Phenom. 1999, 101-103, 309; Phys. Rev. B 1998, $57,6258$.

4. Beaurepaire, E.; Maret, M.; Halté, V.; Merle, J.-C.; Daunois, A.; Bigot, J. Y. Phys. Rev. B 1998, 58, 12134.

97 Foreword

B. Carrière

98 Organic materials with optical properties

D. Guillon, J.-L. Gallani, L. Mager, S. Méry, J. Le Moigne, D. Navarro-Rodriguez and J.-F. Nierengarten

103 Structural and spectroscopic study of organic-inorganic transition metal based layered magnets P. Rabu, M. Drillon and C. Hornick

109 Aggregates in silica based matrices O. Cintora-Gonzalez, C. Estournès, J.-L. Guille, J.-J. Grob, B. Hönerlage, J. Lemoigne, R. Lévy, T. Lutz, J.-C. Merle, D. Muller, M. Richard, J.-L. Rehspringer, J. Schell and N. Viart

114 From random to self-organized clusters on surfaces: a variable temperature STM study S. Padovani, I. Chado, F. Scheurer and J.-P. Bucher

118 Magnetic ultrathin films and multilayers: growth and characterization J.-P. Deville, A. Barbier, C. Mocuta, G. Renaud, Y. Samson, H. Magnan, P. Le Fèvre, D. Chandesris, P. Panissod and C. Mény

Cover pictures:

Top: Magnetic organic-inorganic multilayers (P. Rabu).

Middle: Photovoltaic fullerene derivative (D. Guillon)

Bottom: STM image of self-assembled cobalt clusters (S. Padovani) 